\title{
Supporting information Atomically Embedded Ag via Electro-diffusion Boosts Oxygen Evolution of CoOOH Nanosheet Arrays
}

\author{
Changsoo Lee ${ }^{1 \ddagger}$, Kihyun Shin ${ }^{2 \ddagger}$, Chanwon Jung ${ }^{1 \ddagger}$, Pyuck-Pa Choi ${ }^{1}$, Graeme \\ Henkelman $^{2 *}$, and Hyuck Mo Lee ${ }^{1 *}$ \\ ${ }^{1}$ Department of Materials Science and Engineering, KAIST, 291 Daehak-ro, Yuseong- \\ gu, Daejeon, 34141, Republic of Korea \\ ${ }^{2}$ Department of Chemistry, and the Oden Institute for Computational Engineering and \\ Sciences, University of Texas at Austin, Austin, Texas 78712, United States \\ ‡These authors contributed equally to this work. \\ *Corresponding author E-mail: henkelman@utexas.edu, hmlee@,kaist.ac.kr
}

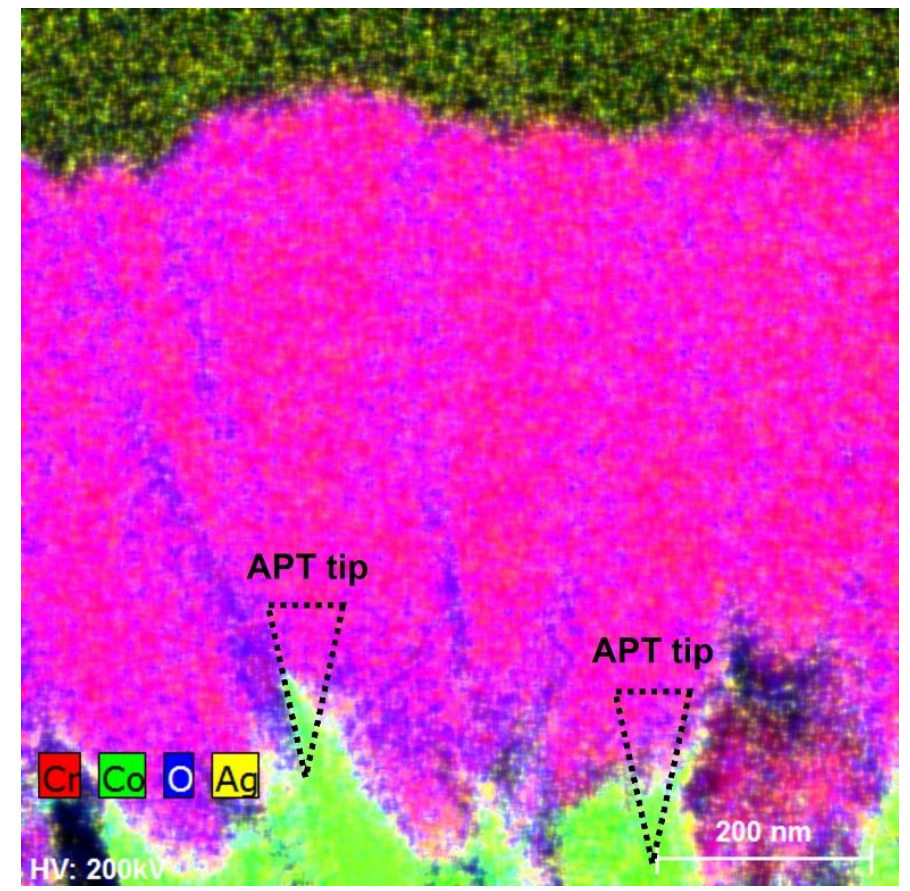

Figure S1. EDS map of cross-section of the Cr-coated Ag-doped $\mathrm{CoOOH}$ electrocatalysts: lift-off of APT tips are described as the dashed lines. 
(a)

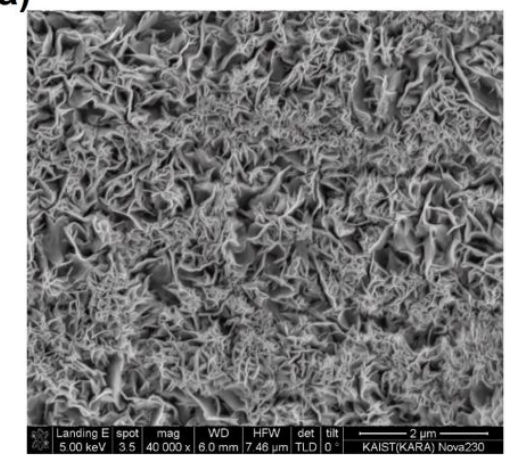

(c)

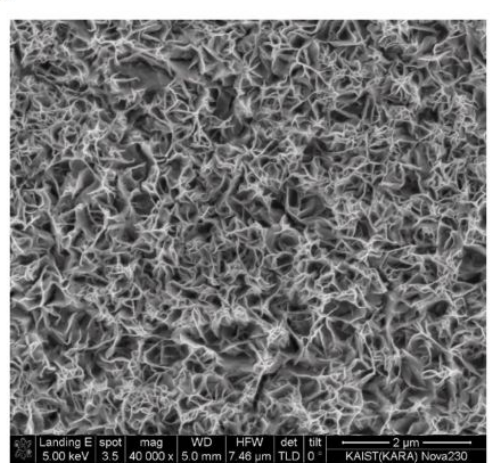

(b)

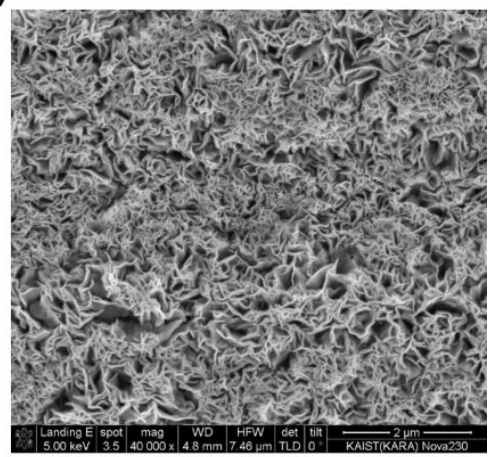

(d)

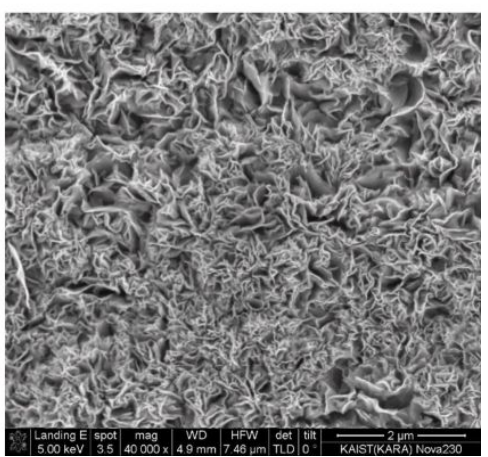

Figure S2. Surface morphologies of $\mathrm{CoOOH}$ on each metallic substrate: (a) $\mathrm{Ag}$, (b) $\mathrm{Au}$, (c) $\mathrm{Cu}$, and (d) $\mathrm{Ni}$.

\section{(a)}

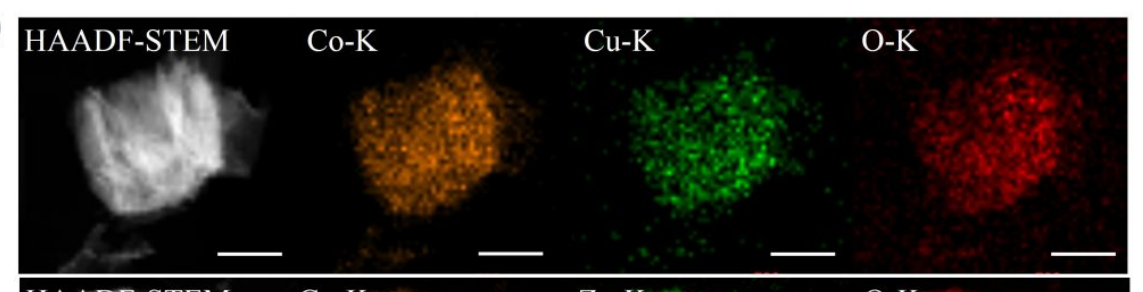

(b)

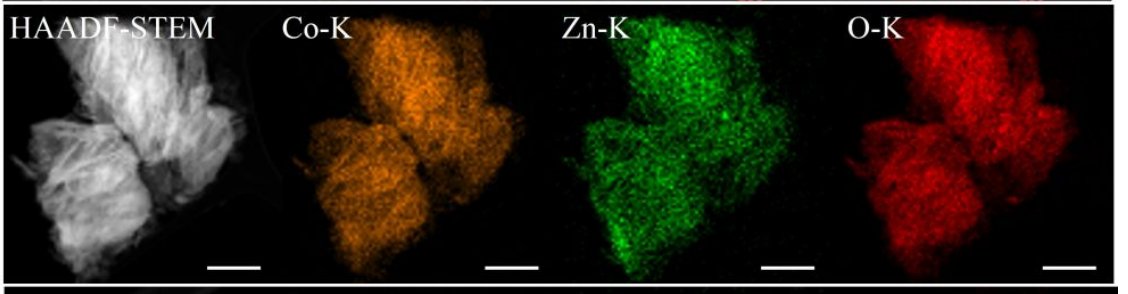

(c)

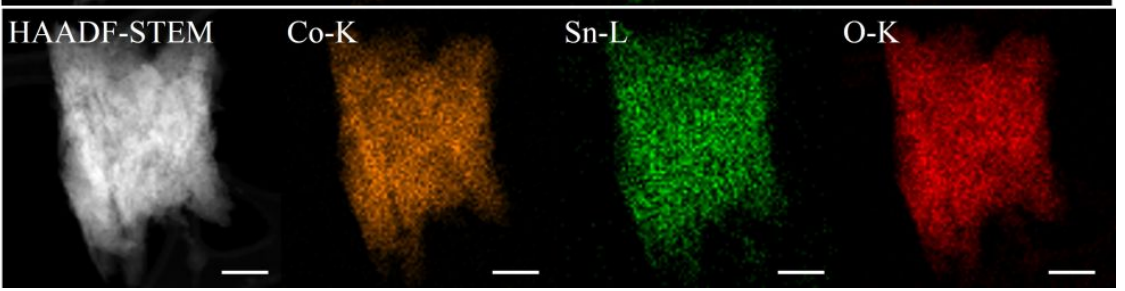

Figure S3. EDS elemental maps of exfoliated $\mathrm{CoOOH}$ on (a) $\mathrm{Cu}$, (b) $\mathrm{Zn}$, and (c) $\mathrm{Sn}$ substrates. Scale bars: (a) $300 \mathrm{~nm}$, (b) $500 \mathrm{~nm}$, and (c) $100 \mathrm{~nm}$. 

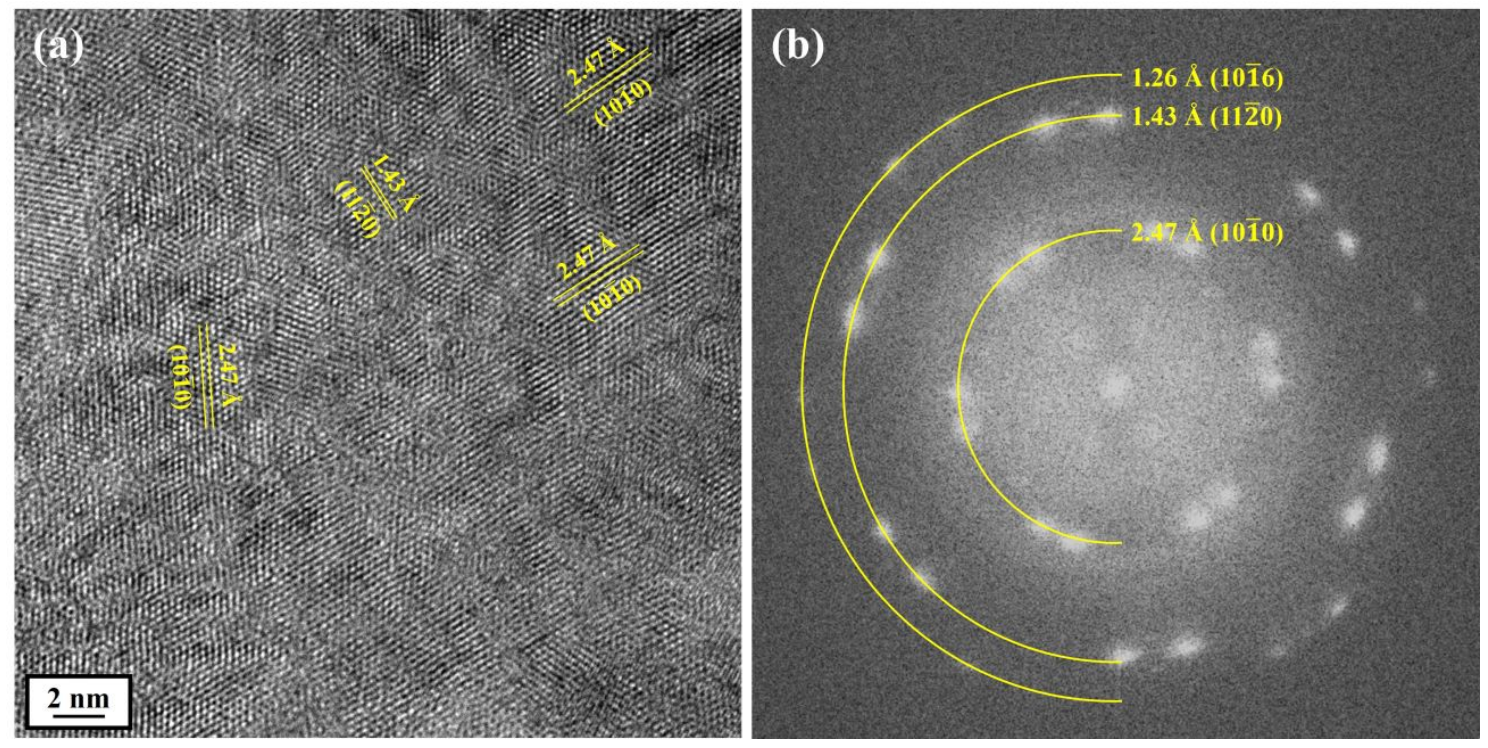

Figure S4. (a) HRTEM of Ag-doped $\mathrm{CoOOH}$, and (b) its fast-Fourier transformed selected area electron diffraction pattern (FFT-SAED).
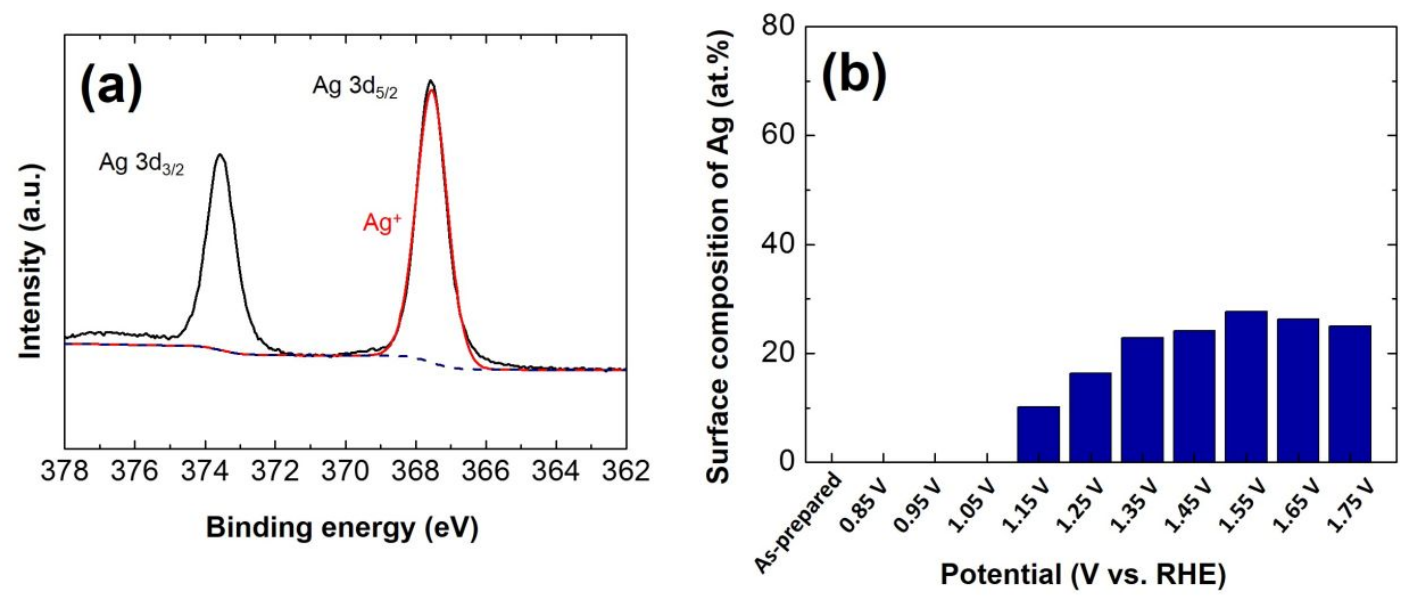

Figure S5. (a) XPS Ag 3d peak, and (b) Quantitative XPS analysis of Ag-doped $\mathrm{CoOOH}$ with applied voltages ranging from $0.85 \mathrm{~V}$ to $1.75 \mathrm{~V}$ (vs. RHE), indicating the surface composition at each voltage. 


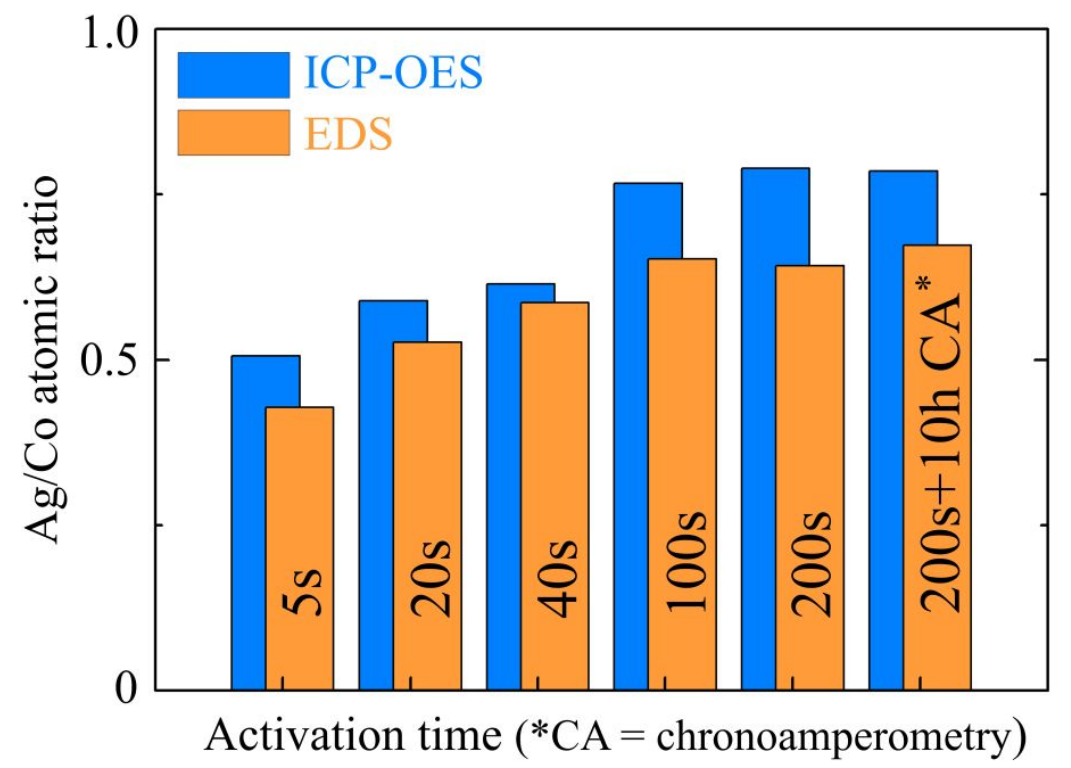

Figure S6. Compositional investigation of Ag diffusions at $1.4 \mathrm{~V}$ (vs. RHE) with EDS and ICP-OES compositional measurements; $10 \mathrm{~h} \mathrm{CA}$ was conducted at the overpotential of $300 \mathrm{mV}$; the compositions were measured with exfoliated electrocatalysts using ultra-sonication for $30 \mathrm{~min}$.

(a) $\mathrm{co}_{\mathrm{o}}$

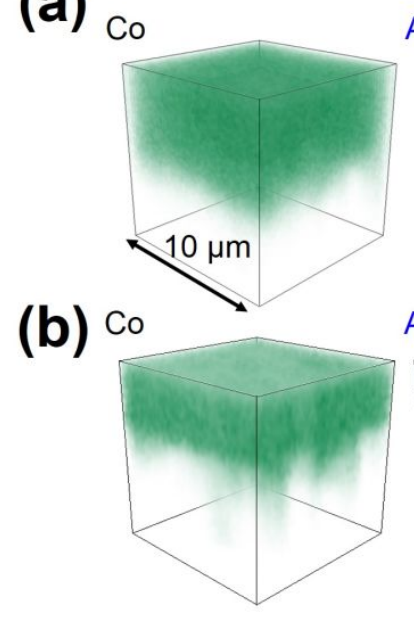

$\mathrm{Ag}$

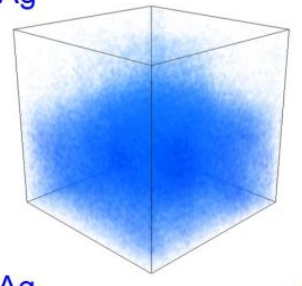

$\mathrm{Ag}$

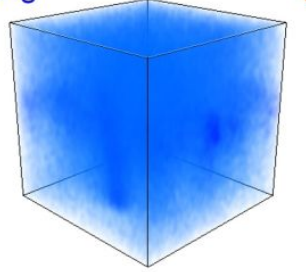

$\mathrm{O}$

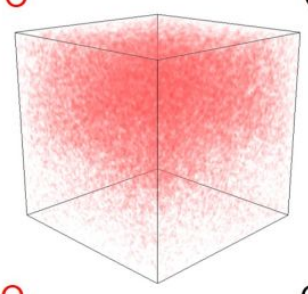

$\mathrm{O}$

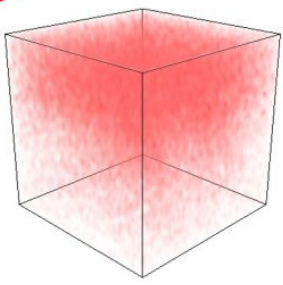

Overlay

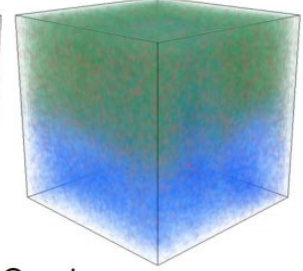

Overlay

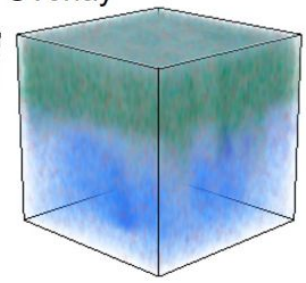

(c)

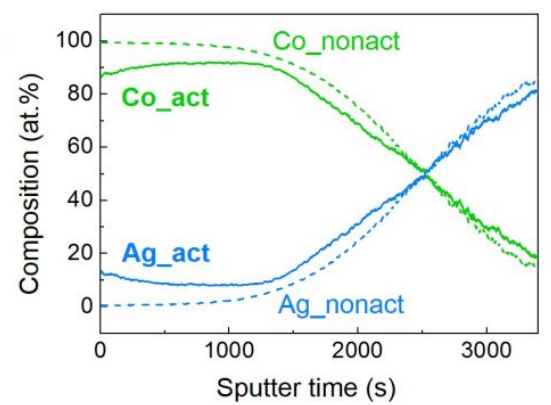

Figure S7. SIMS 3D rending of each element and overlay for Ag-doped $\mathrm{CoOOH}$ : (a) before activation, and (b) after activation; (c) their depth profiling. 


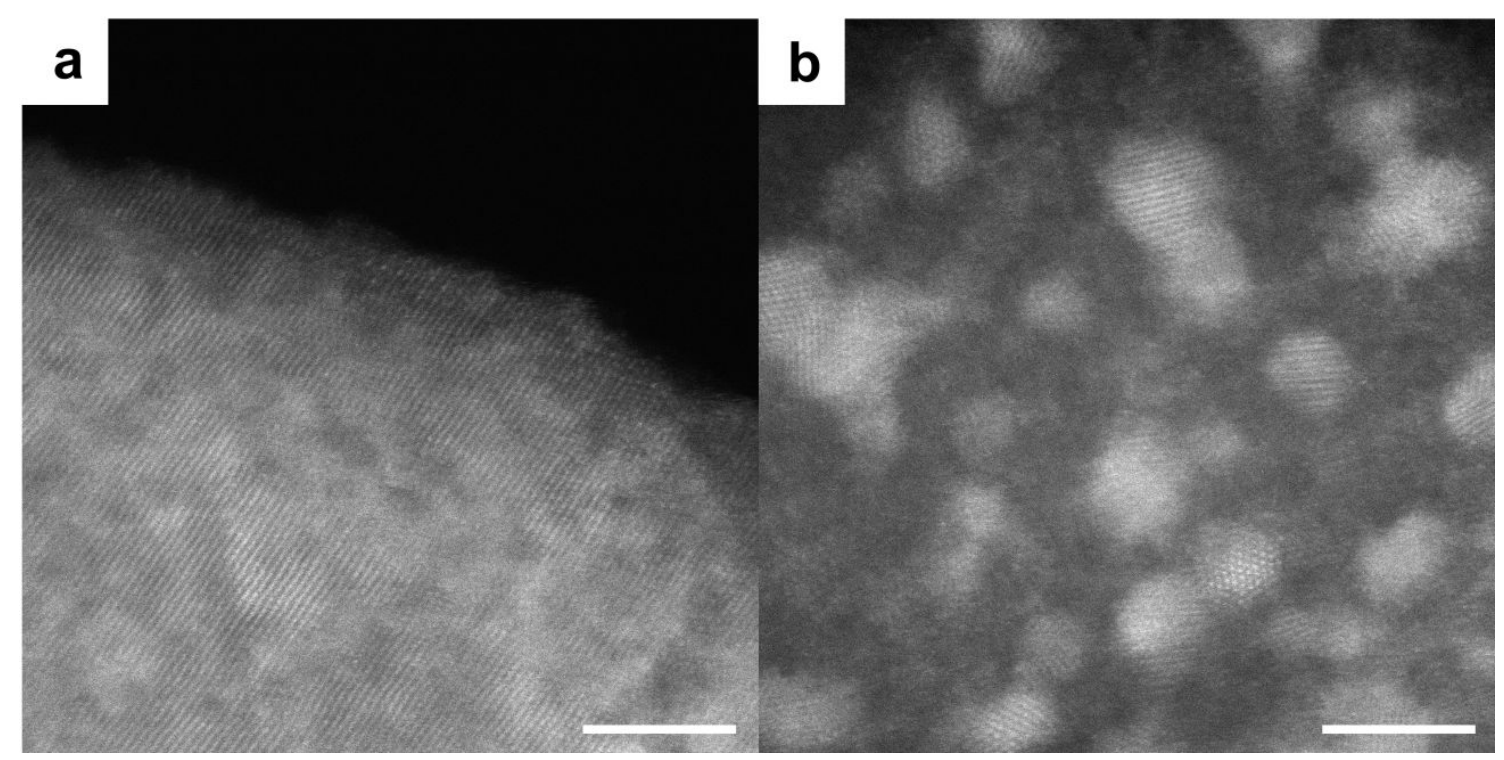

Figure S8. HADDF-STEM images of (a) Ag-doped $\mathrm{CoOOH}$, and (b) $\mathrm{Ag}_{2} \mathrm{O} / \mathrm{CoOOH}$ after heat-treatment in $80^{\circ} \mathrm{C}$ for $10 \mathrm{~h}$. Scale bars: (a) and (b) $5 \mathrm{~nm}$.

(a)

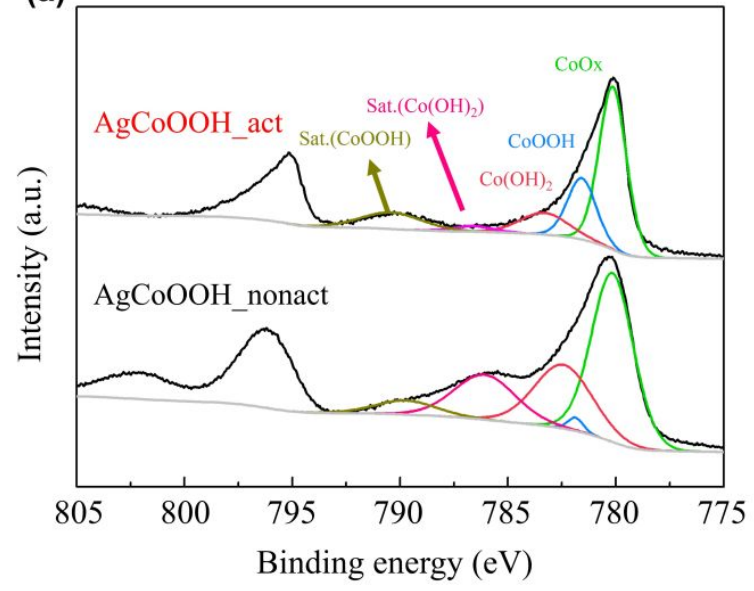

(b)

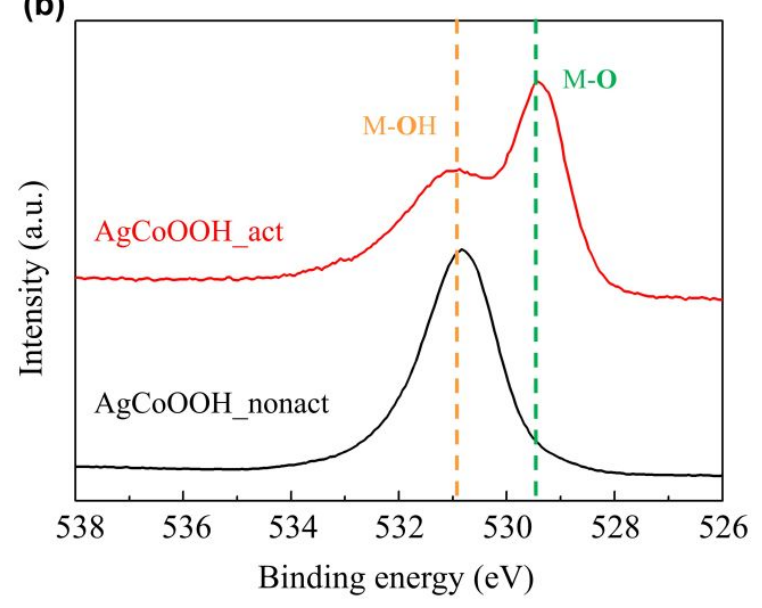

Figure S9. (a) XPS Co 2p peaks, and (b) O 1s peaks; for $\mathrm{CoOOH}$ on $\mathrm{Ag}$ before and after activation. 
(a)

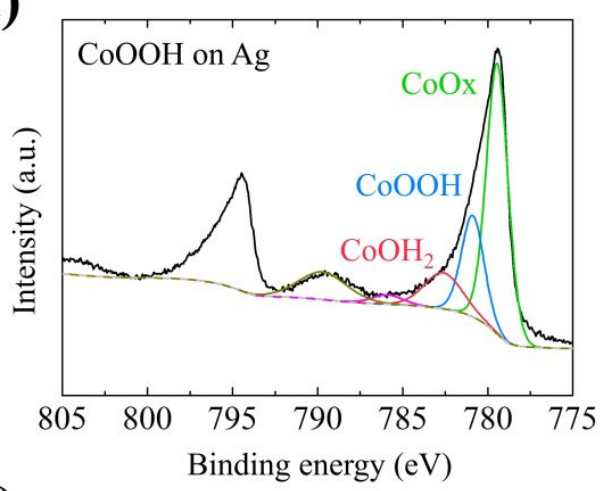

(c)

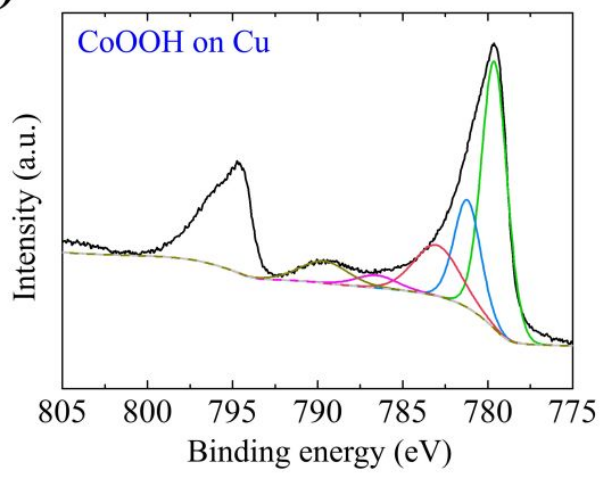

(b)

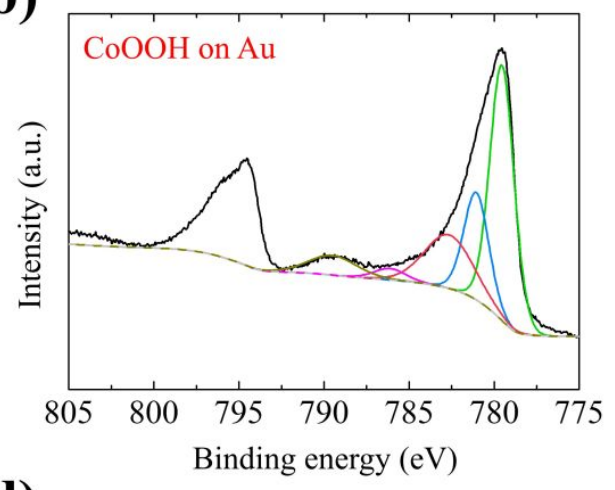

(d)

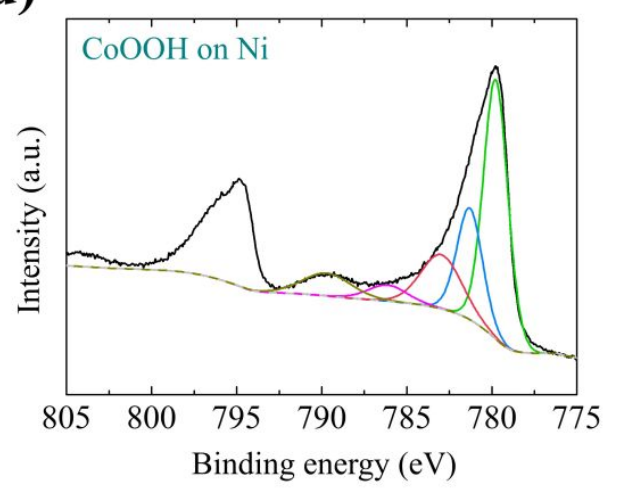

Figure S10. XPS Co 2p peaks for $\mathrm{CoOOH}$ on (a) $\mathrm{Ag}$, (b) $\mathrm{Au},(\mathbf{c}) \mathrm{Cu}$, and (d) $\mathrm{Ni}$, after the activation process.

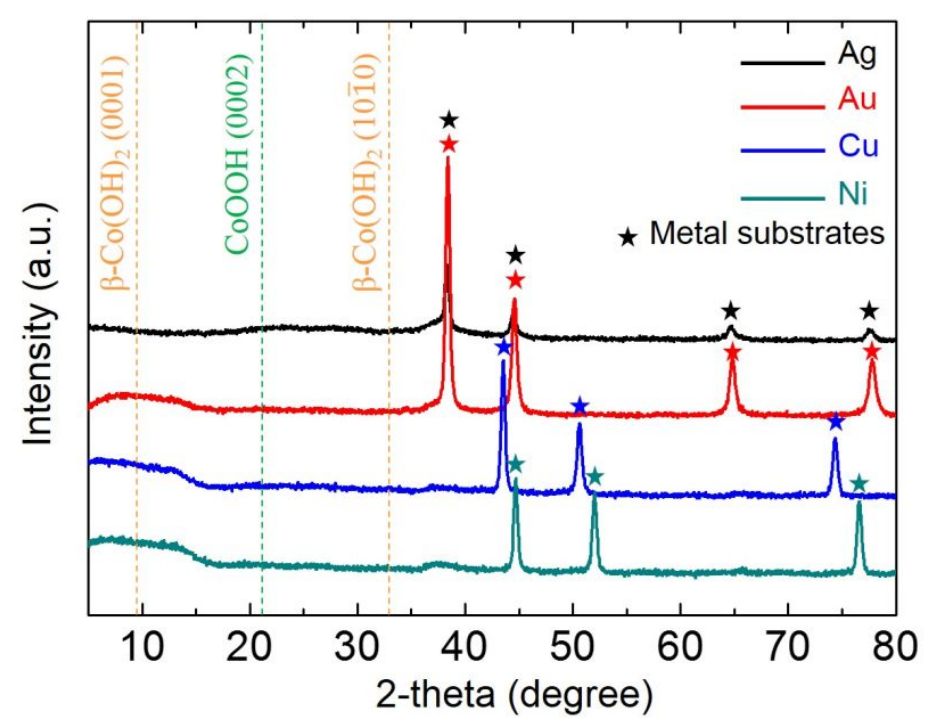

Figure S11. XRD analysis of $\mathrm{CoOOH}$ on $\mathrm{Ag}, \mathrm{Au}, \mathrm{Cu}$, and Ni; ICDD No.00-051-1731 and No.01-072-2280 for $\mathrm{Co}(\mathrm{OH})_{2}$ and $\mathrm{CoOOH}$, respectively. 


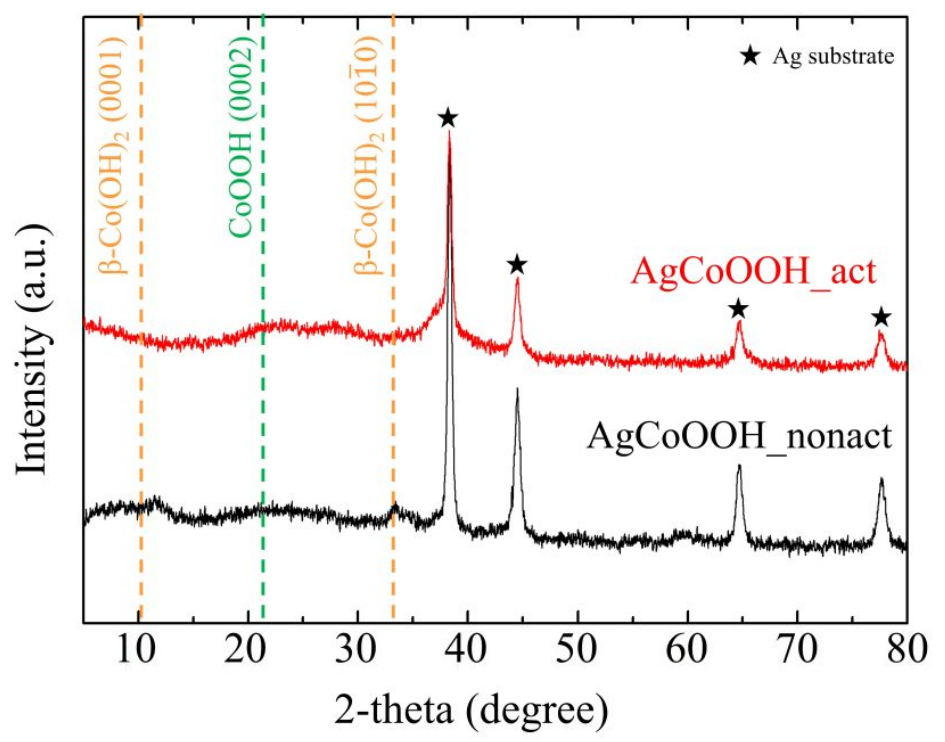

Figure S12. XRD analysis of Ag-doped $\mathrm{CoOOH}$ before and after activation; ICDD No.00-051-1731 and No.01-072-2280 for $\mathrm{Co}(\mathrm{OH})_{2}$ and $\mathrm{CoOOH}$, respectively.

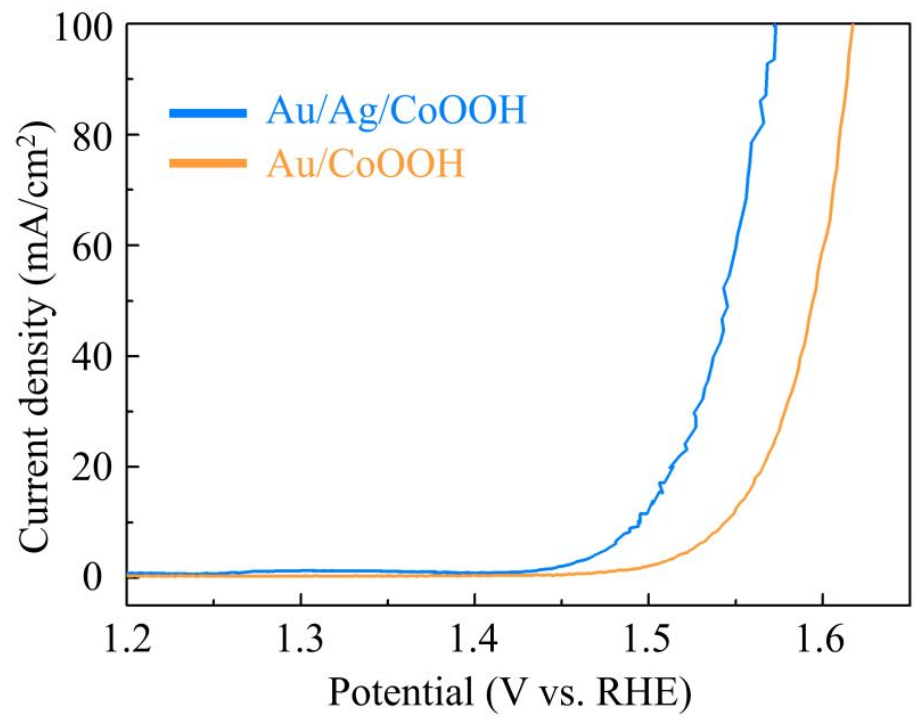

Figure S13. OER polarization curves of $\mathrm{CoOOH}$ on $\mathrm{Au}$, and $\mathrm{CoOOH}$ on $\mathrm{Ag}$ electrodeposited Au substrate; Ag was deposited on Au substrate using a two-step chronoamperometry process $\left(1 \mathrm{~mA} / \mathrm{cm}^{2}\right.$ for $100 \mathrm{~s}$ and $10 \mathrm{~mA} / \mathrm{cm}^{2}$ for $\left.60 \mathrm{~s}\right)$. 


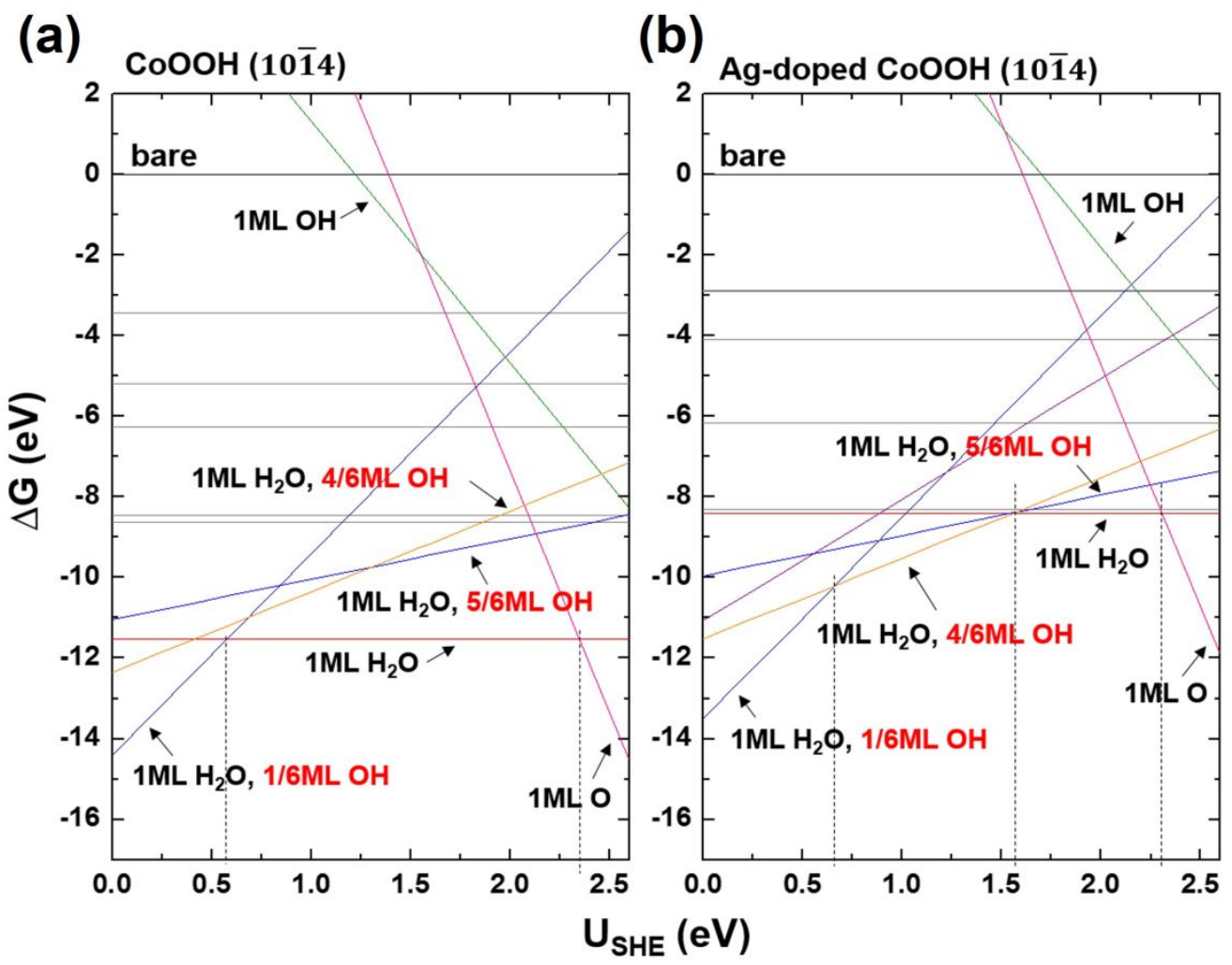

Figure S14. Surface free energy diagram of (a) $\mathrm{CoOOH}(10 \overline{1} 4)$, and (b) Ag-doped $\mathrm{CoOOH}$ (1014). 
(a)

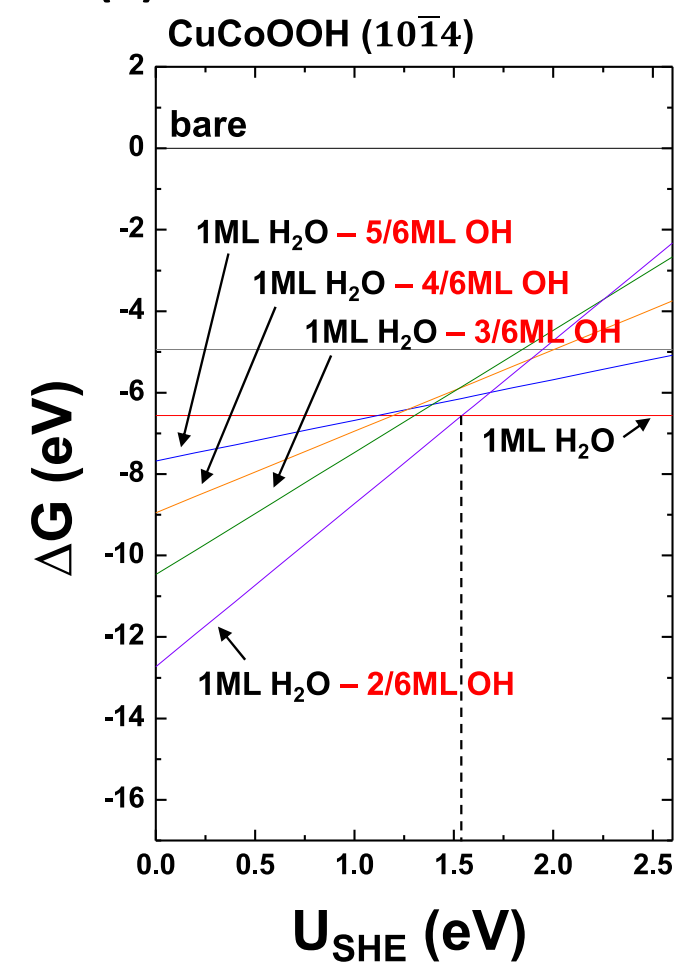

(b)

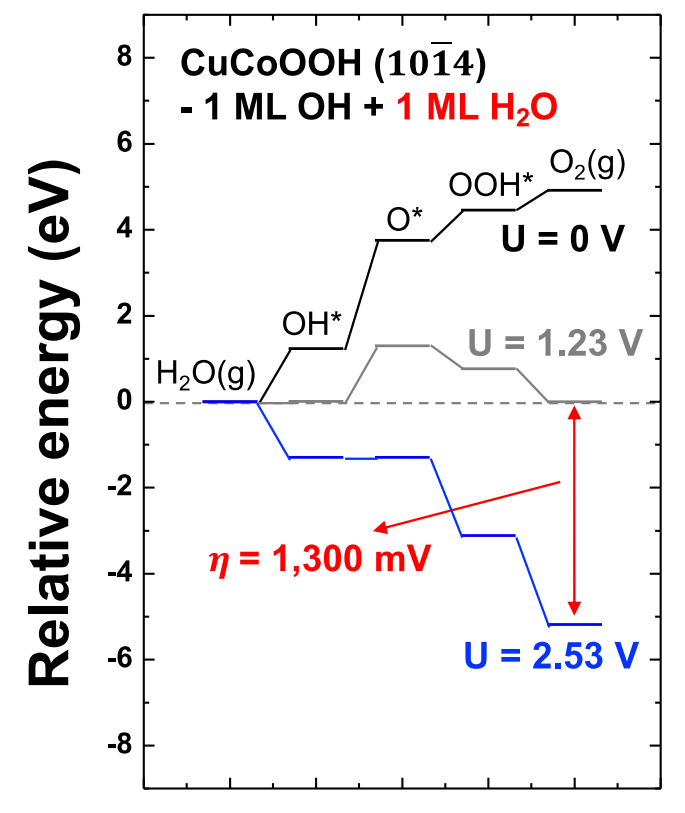

Reaction coordinates

Figure S15. Surface free energy diagram of (a) $\mathrm{Cu}$-doped $\mathrm{CoOOH}(10 \overline{1} 4)$, and (b) Gibbs free energy diagram with OER overpotential for $\mathrm{Cu}$-doped $\mathrm{CoOOH}(10 \overline{1} 4)$.

Table S1. EDS compositions of exfoliated $\mathrm{CoOOH}$ on $\mathrm{Ag}, \mathrm{Au}, \mathrm{Cu}$ and $\mathrm{Ni}$.

\begin{tabular}{l|c|c|c} 
& Co (at.\%) & X (at.\%) & O (at.\%) \\
\hline CoOOH on Ag & 20.33 & 13.22 & 66.44 \\
$\mathrm{CoOOH}$ on $\mathrm{Au}$ & 31.93 & - & 68.07 \\
$\mathrm{CoOOH}$ on $\mathrm{Cu}$ & 32.60 & 9.31 & 58.09 \\
$\mathrm{CoOOH}$ on $\mathrm{Ni}$ & 26.86 & - & 73.14
\end{tabular}

TEM EDS composition (10 points average) 
Table S2. Recently reported Co-based electrocatalysts and their OER performances

\begin{tabular}{cccc}
\hline Catalysts & Electrodes & $\begin{array}{c}\text { Overpotentials } \\
(\mathbf{m V}) @ \mathbf{~} \mathbf{1 0} \\
\mathbf{m A} \mathbf{c} \mathbf{c m}^{\mathbf{2}}\end{array}$ & References \\
\hline $\mathrm{CeO}_{\mathrm{x}} / \mathrm{CoO}_{\mathrm{x}}$ & $\mathrm{Ti}$ & $\mathbf{3 1 3}$ & $\mathbf{1}$ \\
$\mathrm{Co} \mathrm{doped} \mathrm{CuS}$ & $\mathrm{GC}$ & $\mathbf{2 7 0}$ & $\mathbf{2}$ \\
$\mathrm{Ag} @ \mathrm{Co}_{\mathrm{x}} \mathrm{P}$ & $\mathrm{GC}$ & $\mathbf{3 1 0}$ & $\mathbf{3}$ \\
$\mathrm{NiCo}_{2} \mathrm{O}_{4}$ & $\mathrm{Ni}$ foam & $\mathbf{2 9 0}$ & $\mathbf{4}$ \\
$\mathrm{CoNi}(\mathrm{OH})_{\mathrm{x}}$ & $\mathrm{Cu}$ foils & $\mathbf{2 8 0}$ & $\mathbf{5}$ \\
$\mathrm{Co}-\mathrm{defected} \mathrm{Co}{ }_{3-\mathrm{x}} \mathrm{O}_{4}$ & $\mathrm{GC}$ & $\mathbf{2 6 8}$ & $\mathbf{6}$ \\
$\left(\mathrm{Co}{ }_{1-\mathrm{x}} \mathrm{Fe}\right)_{2} \mathrm{P}$ & - & $\mathbf{2 7 0}$ & $\mathbf{7}$ \\
$\mathrm{CoSn}(\mathrm{OH})_{6}$ & $\mathrm{GC}$ & $\mathbf{2 7 4}$ & $\mathbf{8}$ \\
$\mathrm{NiCoP} \mathrm{NWs}$ & $\mathrm{GC}$ & $\mathbf{2 2 1}$ & $\mathbf{9}$ \\
$\mathrm{Fe}-\mathrm{CoP} / \mathrm{CoO}$ & $\mathrm{GC}$ & $\mathbf{2 1 9}$ & $\mathbf{1 0}$ \\
$\mathrm{Co}(\mathrm{OH})_{2} @ \mathrm{~g}_{-} \mathrm{C}_{3} \mathrm{~N}_{4}$ & $\mathrm{GC}$ & $\mathbf{3 2 0}$ & $\mathbf{1 1}$ \\
\hline
\end{tabular}

\section{References}

1. Kim, J. H.; Shin, K.; Kawashima, K.; Youn, D. H.; Lin, J.; Hong, T. E.; Liu, Y.; Wygant, B. R.; Wang, J.; Henkelman, G.; Mullins, C. B., Enhanced Activity Promoted by $\mathrm{CeO}_{\mathrm{x}}$ on a $\mathrm{CoO}_{\mathrm{x}}$ Electrocatalyst for the Oxygen Evolution Reaction. $A C S$ Catal. 2018, 8, 4257-4265.

2. $\quad$ Li, Q.; Wang, X. F.; Tang, K.; Wang, M. F.; Wang, C.; Yan, C. L., Electronic Modulation of Electrocatalytically Active Center of $\mathrm{Cu}_{7} \mathrm{~S}_{4}$ Nanodisks by Cobalt-Doping for Highly Efficient Oxygen Evolution Reaction. ACS Nano 2017, 11, 12230-12239.

3. Hou, Y. H.; Liu, Y. P.; Gao, R. Q.; Li, Q. J.; Guo, H. Z.; Goswami, A.; Zboril, R.; Gawande, M. B.; Zou, X. X., Ag@Cox P Core-Shell Heterogeneous Nanoparticles as Efficient Oxygen Evolution Reaction Catalysts. ACS Catal. 2017, 7, 7038-7042.

4. Gao, X. H.; Zhang, H. X.; Li, Q. G.; Yu, X. G.; Hong, Z. L.; Zhang, X. W.; Liang, C. D.; Lin, Z., Hierarchical $\mathrm{NiCo}_{2} \mathrm{O}_{4}$ Hollow Microcuboids as Bifunctional Electrocatalysts for Overall Water-Splitting. Angew. Chem. Int. Edit. 2016, 55, 62906294.

5. Li, S. W.; Wang, Y. C.; Peng, S. J.; Zhang, L. J.; Al-Enizi, A. M.; Zhang, H.; Sun, X. H.; Zheng, G. F., Co-Ni-Based Nanotubes/Nanosheets as Efficient Water Splitting Electrocatalysts. Adv. Energy Mater. 2016, 6, 1501661.

6. Zhang, R. R.; Zhang, Y. C.; Pan, L.; Shen, G. Q.; Mahmood, N.; Ma, Y. H.; Shi, Y.; Jia, W. Y.; Wang, L.; Zhang, X. W.; Xu, W.; Zou, J. J., Engineering Cobalt Defects in Cobalt Oxide for Highly Efficient Electrocatalytic Oxygen Evolution. ACS 
Catal. 2018, 8, 3803-3811.

7. Tan, Y. W.; Wang, H.; Liu, P.; Shen, Y. H.; Cheng, C.; Hirata, A.; Fujita, T.; Tang, Z.; Chen, M. W., Versatile Nanoporous Bimetallic Phosphides towards Electrochemical Water Splitting. Energ. Environ. Sci. 2016, 9, 2257-2261.

8. Song, F.; Schenk, K.; Hu, X. L., A Nanoporous Oxygen Evolution Catalyst Synthesized by Selective Electrochemical Etching of Perovskite Hydroxide $\mathrm{CoSn}(\mathrm{OH})_{6}$ Nanocubes. Energ. Environ. Sci. 2016, 9, 473-477.

9. Xu, H.; Wei, J. J.; Zhang, M.; Wang, J.; Shiraishi, Y.; Tian, L.; Du, Y., SelfSupported Nickel-Cobalt Nanowires as Highly Efficient and Stable Electrocatalysts for Overall Water Splitting. Nanoscale 2018, 10, 18767-18773.

10. Hu, X. M.; Zhang, S. L.; Sun, J. W.; Yu, L.; Qian, X. Y.; Hu, R. D.; Wang, Y. N.; Zhao, H. G.; Zhu, J. W., 2D Fe-Containing Cobalt Phosphide/Cobalt Oxide Lateral Heterostructure with Enhanced Activity for Oxygen Evolution Reaction. Nano Energy 2019, 56, 109-117.

11. Tahir, M.; Mahmood, N.; Pan, L.; Huang, Z. F.; Lv, Z.; Zhang, J. W.; Butt, F. K.; Shen, G. Q.; Zhang, X. W.; Dou, S. X.; Zou, J. J., Efficient Water Oxidation through Strongly Coupled Graphitic $\mathrm{C}_{3} \mathrm{~N}_{4}$ Coated Cobalt Hydroxide Nanowires. $J$. Mater. Chem. A 2016, 4, 12940-12946. 